OPEN ACCESS

Edited by:

Torben Moos,

Aalborg University,

Denmark

Reviewed by:

Ruben Vidal,

Indiana University School of

Medicine

USA

Kasper Bendix Johnsen,

Aalborg University,

Denmark

*Correspondence:

Franca Codazzi and Fabio Grohovaz, Division of Neuroscience, San Raffaele Scientific Institute and University, via Olgettina 58, Milano 20132, Italy codazzi.franca@hsr.it; grohovaz.fabio@hsr.it

Received: 04 August 2014 Accepted: 19 May 2015 Published: 03 June 2015

Citation:

Codazzi F, Pelizzoni I, Zacchetti D and Grohovaz F (2015) Iron entry in neurons and astrocytes: a link with synaptic activity.

Front. Mol. Neurosci. 8:18. doi: 10.3389/fnmol.2015.00018

\section{Iron entry in neurons and astrocytes: a link with synaptic activity}

\author{
Franca Codazzi ${ }^{1,2 *}$, Ilaria Pelizzoni ${ }^{2}$, Daniele Zacchetti ${ }^{2}$ and Fabio Grohovaz ${ }^{1,2 *}$ \\ ${ }^{1}$ Vita-Salute San Raffaele University, Milan, Italy, ${ }^{2}$ Division of Neuroscience, San Raffaele Scientific Institute and University, \\ Milan, Italy
}

Iron plays a fundamental role in the development of the central nervous system (CNS) as well as in several neuronal functions including synaptic plasticity. Accordingly, neuronal iron supply is tightly controlled: it depends not only on transferrin-bound iron but also on non-transferrin-bound iron (NTBI), which represents a relevant quote of the iron physiologically present in the cerebrospinal fluid (CSF). Different calcium permeable channels as well as the divalent metal transporter 1 (DMT1) have been proposed to sustain NTBI entry in neurons and astrocytes even though it remains an open issue. In both cases, it emerges that the control of iron entry is tightly linked to synaptic activity. The iron-induced oxidative tone can, in physiological conditions, positively influence the calcium levels and thus the synaptic plasticity. On the other hand, an excess of iron, with the ensuing uncontrolled production of reactive oxygen species (ROS), is detrimental for neuronal survival. A protective mechanism can be played by astrocytes that, more resistant to oxidative stress, can uptake iron, thereby buffering its concentration in the synaptic environment. This competence is potentiated when astrocytes undergo activation during neuroinflammation and neurodegenerative processes. In this minireview we focus on the mechanisms responsible for NTBI entry in neurons and astrocytes and on how they can be modulated during synaptic activity. Finally, we speculate on the relevance they may have in both physiological and pathological conditions.

Keywords: iron, neurons, astrocytes, oxidative stress, synapses

\section{Introduction}

Iron transport in cells and body fluids requires the participation of specific proteins and buffering molecules to avoid precipitation of the metal ion, with the potential induction of cytotoxic effects. In the plasma, the main binding protein is transferrin (Tf), even though heme and ferritin can contribute to bind and circulate iron. In particular, iron can be transported as hemoglobin bound to haptoglobin as well as heme directly bound to hemopexin. On the other hand, ferritin iron represents only a minute amount of the circulating iron (Crichton and Charloteaux-Wauters, 1987; Brissot et al., 2012). Plasma iron concentration can exceed the total binding capacity of Tf only in conditions of severe pathological iron overload, as it occurs, for instance, in different forms of hemochromatosis (Janssen and Swinkels, 2009). Along with the transferrin-bound iron, another iron pool was identified by Hershko et al. (1978) and called non-transferrin-bound iron (NTBI). However this definition is somewhat confusing since NTBI does not include all forms of iron unbound to transferrin; for instance heme and ferritin iron are excluded (Brissot et al., 2012). Plasmatic NTBI is composed mainly by Fe(III), loosely bound to buffering molecules (mainly citrate and acetate), but also to the abundant plasma 
protein, albumin (Hider, 2002; Brissot et al., 2012). This pool has attracted great interest since it may represent a relevant source of iron for various cell types (e.g., in hepatocytes; Wang and Knutson, 2013) and can have a pathological relevance (e.g., in cardiomiocytes; Oudit et al., 2006).

Different is the situation of iron homeostasis in the central nervous system (CNS): although the mechanisms are still debated and somewhat controversial, a large body of evidence indicates that the iron control in CNS is virtually independent of the rest of the body. Indeed, conditions of systemic iron overload or deficiency are reported not to significantly affect the brain (Russo et al., 2004; Moos et al., 2007). Moreover, also the role and the contribution of transferrin-bound iron and NTBI to iron homeostasis appear clearly different in the two compartments. In fact, transferrin-bound iron is reported to operate with the same mechanisms described at the systemic level in neurons but not in glial cells. On the other hand, NTBI is attracting more attention in light of the various mechanisms that are potentially involved and of the possible regulation they are subjected to during synaptic activity (see next chapter). Based on this premise, we will focus our attention on NTBI, its composition and the mechanisms sustaining its uptake in brain cells.

\section{NTBI in the Brain}

Brain shows clear differences in the control of iron homeostasis that can account for its specific physiopathological behavior. Despite the difficulty of measuring iron concentration within the cerebrospinal fluid (CSF) and brain interstitium, a value much lower than in the plasma was estimated $(0.25-1 \mu \mathrm{M}$ vs. $\sim 20 \mu \mathrm{M})$. Considering that the concentration of the Tf produced in the brain (by the choroid plexuses and oligodendrocytes) is more than two orders of magnitude lower than in the plasma (i.e., $\sim 0.2 \mu \mathrm{M}$ vs. $\sim 30 \mu \mathrm{M}$ ), Tf is expected to be fully saturated in the CSF. Accordingly, NTBI significantly contributes to total iron in CSF and brain interstitium even in physiological conditions (Molina et al., 1998; Moos and Morgan, 1998; Mizuno et al., 2005; Hozumi et al., 2011). Moreover, the presence of a very high concentration of ascorbate in the CSF (up to 100 times, compared to plasma; Bradbury, 1997; Lane et al., 2010), generates a reducing environment that increases the ratio $\mathrm{Fe}(\mathrm{II}) / \mathrm{Fe}(\mathrm{III})$ in NTBI. The expression of different ferric reductases (duodenal cytochrome B, Dcytb, and stromal cellderived receptor 2, SDR2) further contributes to make Fe(II) the prominent form of iron in the NTBI (Tulpule et al., 2010). This is particularly relevant since Fe(II) represents the main form of iron acquirable by astrocytes, which do not have Tf receptors, but also an important source for neurons (Moos et al., 2007; Pelizzoni et al., 2011, 2013). However, NTBI has not only a relevant physiological role in the CNS but it can acquire a pathological relevance, as suggested by growing evidence that NTBI levels increase during aging as well as in several neurodegenerative disorders (Bradbury, 1997; Breuer et al., 2000; Crichton et al., 2011). From this standpoint, the NTBI can be related to the onset and the progression of neurodegenerative disorders, not necessarily related to genetic alteration of iron homeostasis. Therefore, a thorough characterization of the cellular pathways and of the molecular mechanisms controlling NTBI in the brain can open new vistas on the etiopathogenesis of many neurodegenerative diseases.

\section{Mechanisms of NTBI Uptake by Neurons and Astrocytes}

A number of questions are still open about the mechanisms of NTBI entry in brain-derived cells: which are the pathways that operate in neurons and astrocytes? Which is their relevance in specific brain regions? Can neuronal functions and, in particular, synaptic activity, influence NTBI uptake? And, finally, can pathological conditions affect these pathways?

NTBI uptake was first proposed in cortical neurons (Cheah et al., 2006), where a role for divalent metal transporter 1 (DMT1), the main iron transporter expressed in mammalian cells (Gunshin et al., 1997), was reported. In particular, it was suggested that a calcium influx mediated by the glutamatergic $\mathrm{N}$-methyl-D-aspartate receptor (NMDAR) is able to promote a positive modulation of the transporter, and thus of $\mathrm{Fe}$ (II) uptake, as a consequence of its interaction with Dexras1 (a member of the Ras family) via an adaptor protein. However no evidence of a direct involvement of DMT1 in the iron influx was provided; moreover, data were not obtained in physiological conditions, since the experiments were performed at a $\mathrm{pH}$ of 5.5. This paper thus proposes, for the first time, a link between synaptic activity and iron entry in neuronal cells. This general concept also emerged in a following study from Hidalgo's group (Haeger et al., 2010) in which it was reported that a brief stimulation with NMDA was sufficient to promote the transcription of the isoform $1 \mathrm{~B} / \mathrm{IRE}(+)$ of DMT1 in hippocampal neurons; however, in this paper, iron entry was not evaluated. Of note, when DMT1-1B/IRE(+) was overexpressed in epithelial cell lines as well as in primary neurons, it was not localized in the recycling endosome, but in the late endosomes/lysosomes, and it was not involved in iron uptake (Yanatori et al., 2010; Pelizzoni et al., 2012). As an alternative, it was proposed that the $\mathrm{Fe}$ (II) may enter neurons directly through $\mathrm{Ca}^{2+}$-permeable channels and, in particular, voltage-operated calcium channels (VOCCs) and NMDARs (Pelizzoni et al., 2011). This mechanism, which might regard all VOCCs but not all $\mathrm{Ca}^{2+}$-permeable channels, involves the competition between $\mathrm{Ca}^{2+}$ and $\mathrm{Fe}$ (II) for the same entry pathway (Lopin et al., 2012), a condition also described in neuronal cell lines (Gaasch et al., 2007) These results are in accordance with those obtained on a model of iron-overloaded cardiomyocytes (Oudit et al., 2003), in which the L-type VOCCs represent the main mechanisms of $\mathrm{Fe}$ (II) entry and thus, of iron-induced cardiomyopathy. The possibility that neurons from different brain areas might differ for the mechanisms of iron entry remains open. Moreover neurons may also acquire the competence to activate specific pathways when the physiological conditions are altered (see below). 
Also astrocytes show a great complexity of iron control, particularly considering that in these cells $\mathrm{Tf}$ receptor 1 was reported not to be expressed in vivo (Moos and Morgan, 2004; Jeong and David, 2006). Recent studies have proposed that cultured astrocytes are capable of importing both $\mathrm{Fe}(\mathrm{II})$ and $\mathrm{Fe}(\mathrm{III})$ and that in the presence of ascorbate, to reproduce the in vivo reducing conditions, iron accumulation is further enhanced (Lane et al., 2010; Tulpule et al., 2010). In both papers an involvement of DMT1 was proposed, even though additional pathways were not excluded. Overall, DMT1 has received great attention and has conditioned our general view of iron uptake. However, the in vivo expression of DMT1 was reported to be confined to astrocytic perivascular endfeet (Moos and Morgan, 2004). Other mechanisms, such as the zinc transporter Zip14, have been proposed for NTBI uptake in astrocytes, even though their physiological role is still to be established (Bishop et al., 2011).

More recently it was shown that, in physiological conditions, $\mathrm{Fe}(\mathrm{II})$ can enter the hippocampal astrocytes through the transient receptor potential canonical (TRPC) channels, with an irrelevant involvement of DMT1 (Pelizzoni et al., 2013). However, upon exposure to proinflammatory cytokines and ensuing activation, astrocytes expressed the DMT1-1A, i.e., the isoform localized on the apical membrane of enterocytes (Hubert and Hentze, 2002). Accordingly, the de novo expression of this isoform of DMT1 in activated astrocytes was proposed to account for their increased capability to uptake Fe(II), but not Fe(III) (Rathore et al., 2012; Pelizzoni et al., 2013; Urrutia et al., 2013). In light of these results, the discrepancies on the importance of DMT1 in astrocytic Fe(II) uptake might be ascribed to the high variability of the culture conditions that can promote different extents of astrocyte activation (Saura, 2007).

\section{Effects of NTBI on Neurons and Role of Astrocytes}

We have seen that NTBI is a relevant source of iron and that various mechanisms can control its supply to neurons and astrocytes. This contribution is important to restore the pool of iron-sulphur clusters and thus to meet the elevate energy consumption caused by neuronal activity, but also to sustain specific neuronal functions, such as neurotransmitter synthesis. Moreover, NTBI can contribute to the "oxidative tone" which is important for both basal synaptic transmission and long-term potentiation (LTP; Muñoz et al., 2011). In particular, it was reported that the iron-mediated reactive oxygen species (ROS) production is required in hippocampal neurons to promote calcium release from ryanodine receptor after NMDA stimulation, an essential event for a sustained LTP. Therefore, a direct coupling between synaptic activity and iron entry can properly address these requirements. However, this is a doubleedged sword in that iron entry, if not properly controlled, represents a harmful condition, because of the capability of $\mathrm{Fe}(\mathrm{II})$ to catalyze the so-called Fenton reaction, which occurs primarily at the mitochondrial level with production of the highly toxic hydroxyl radical (Halliwell, 2006). Accordingly, an increase in NTBI or a potentiation of the import mechanisms can cause high detrimental effects in neurons. A link between elevation of iron levels and neurodegenerative processes has been proposed for aging as well as in several pathological conditions (Altamura and Muckenthaler, 2009; Crichton et al., 2011). The toxic effects of iron accumulation in brain can be particularly harmful when $\mathrm{Fe}(\mathrm{II})$ enters the neurons through $\mathrm{Ca}^{2+}$-permeable channels since it is not controlled by the same intracellular iron level as it occurs for the Tf-mediated pathway and, possibly, also for DMT1 (Hentze et al., 2010). Moreover, the concomitant influx of iron and $\mathrm{Ca}^{2+}$ can greatly contribute to excitotoxicity in glutamatergic neurons (Dixon et al., 2012; Chen et al., 2013). More in general, conditions of increased synaptic activity, in the presence of elevated iron, can cause iron overload and concur to the development of cytotoxic effects (Pelizzoni et al., 2008), as it was suggested to occur in Alzheimer disease (Altamura and Muckenthaler, 2009). Also a potentiation of the mechanisms of iron import can lead up to this dangerous chain of effects. Indeed, an enhancement of DMT1 expression was reported after induction of excitotoxicity (Huang et al., 2006) or hypoxia (Wang et al., 2010) as well as in animal and cellular models of Parkinson disease (Salazar et al., 2008; Du et al., 2009).

This general framework can be further exacerbated in conditions of acute brain injuries and inflammation, which accompany several neurodegenerative disorders, since local acidification favors the reduction of $\mathrm{Fe}$ (III) to $\mathrm{Fe}(\mathrm{II})$ and potentiates the $\mathrm{H}+/$ metal-ion cotransport activity of DMT1 (Mackenzie et al., 2007).

Within this context, astrocytes also play an important role. They were described as participating in brain iron homeostasis under conditions of iron deprivation, with soluble ceruplasmin, released by the same astrocytes, supporting iron uptake in neurons, even though the mechanisms were not elucidated (Ke et al., 2006). More recently, it has been proposed that they participate in the synaptic activity also by buffering iron. Indeed, astrocytes are endowed with high detoxifying defences that make them more resistant than neurons to oxidative insults (Pelizzoni et al., 2011). Under physiological conditions, and even more in the presence of extracellular iron accumulation, these glial cells might buffer perisynaptic iron by uptaking it via TRPC, thereby actively contributing to lowering synaptic iron load. Of note, the activity of members of the TRPC family is enhanced by decreasing the extracellular $\mathrm{pH}$ (Semtner et al., 2007) to values comparable to those observed when exocytosis of the synaptic vesicle is evoked (Palmer et al., 2003).

Overall, it is temping to envision a model in which an increase in synaptic activity favors iron overload in neurons, a condition that is counteracted by the active iron buffering of astrocytes. This protective role is expected to be enhanced during inflammatory conditions when local acidification and astrocyte activation (Xiong et al., 2008), with de novo expression of the DMT1, further enhance iron buffering. This condition has been recently reported in an experimental model of multiple sclerosis, where a significant increase in DMT1 expression was 


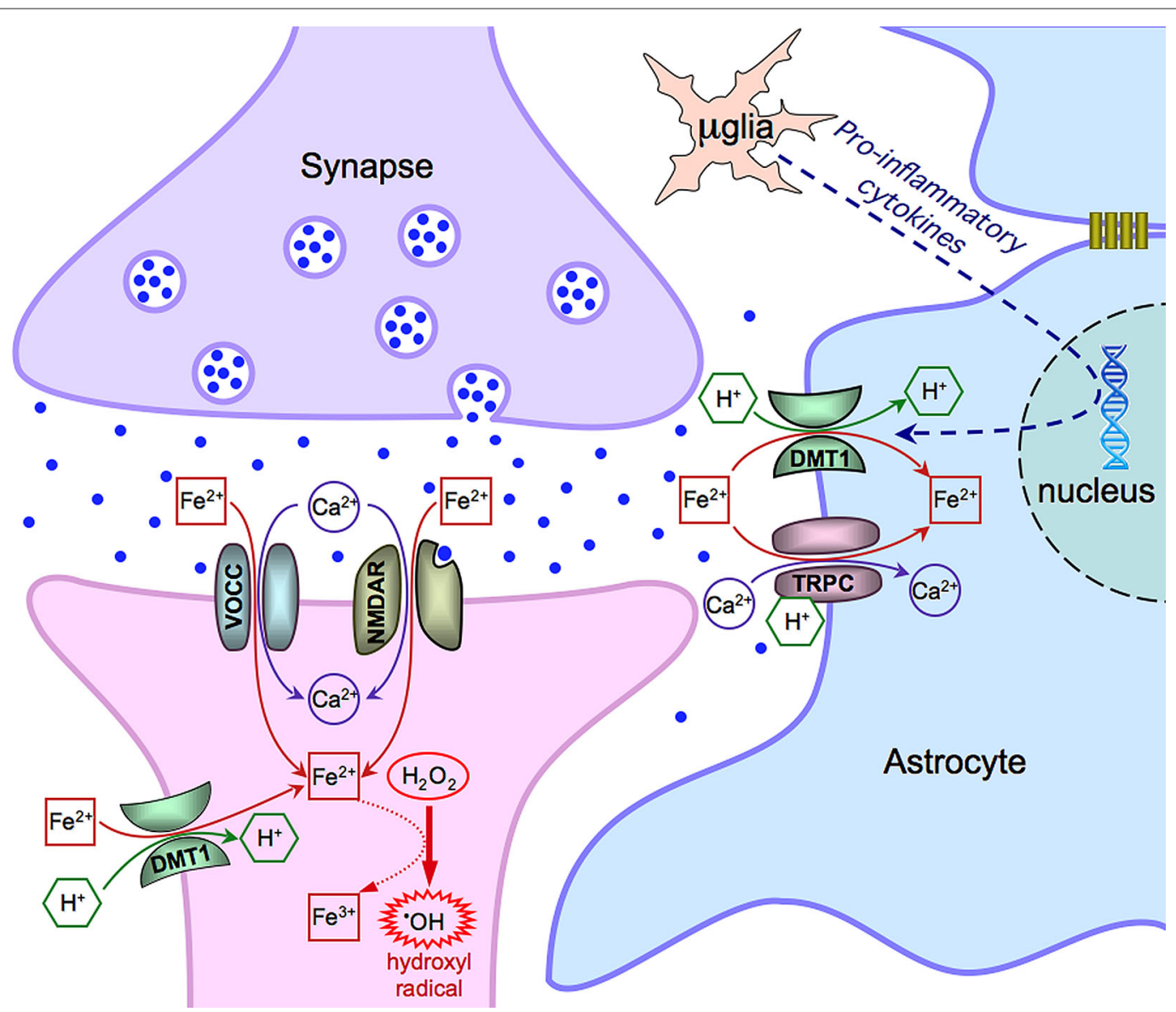

FIGURE 1 | Scheme of the main mechanisms of Fe(II) entry in neurons and astrocytes. Notice that $\mu$ glia indicates a microglia cell. See text for further details and explanations.

observed in astrocytes around the lesions, making them more efficient in recycling iron from the sites of lesions (Zarruk et al., 2015). Of note, inflammatory conditions can cause astrocyte activation with ensuing higher protection against ironmediated oxidative stress (Macco et al., 2013). Conversely, the expression of DMT1 in neurons, as reported in some pathological conditions, might favor iron overload and represent a concause in the neurodegenerative processes.

\section{Conclusions}

Data in the literature propose multiple mechanisms for iron entry in both neurons and astrocytes (summarized in Figure 1) and draw the attention to the role of the neuronal activity on iron influx. In the excitatory synapses of hippocampal neurons, iron uptake depends on the direct stimulation of NMDA receptors and indirect activation of VOCCs (by synaptic activation, back propagating action potentials or depolarization from adjacent synapses). DMT1 can have a physiological role in other brain areas, still showing a dependence on synaptic activity, or can be expressed in some pathological conditions (e.g., Parkinson disease). Within this general framework, iron uptake strongly depends on its availability that can vary with age and in some neurodegenerative diseases (Bradbury, 1997; Molina et al., 1998). In this respect, astrocytes, closely associated to neurons, operate to buffer the excess of extracellular iron. This is important under physiological conditions but, particularly, in pathological conditions when this competence is reinforced by the expression of additional mechanisms of iron uptake. Overall, this complex interplay, including the functional coupling of astrocytes through gap junctions, may help in buffering iron and in protecting neurons during neuroinflammation and neurodegenerative processes.

\section{Acknowledgments}

We thank the other members of the Cellular Neurophysiology Unit at San Raffaele for helpful discussions. This work was conducted within the framework of the Cluster Tecnologico Nazionale Scienze della Vita "Alisei”, Ivascomar project (Italian Ministry of Research). Financial support was from: the Italian Ministry of Research (PRIN project 20108WT59Y) and Telethon Italy (GGP10099 grant) to FG; National Ataxia Foundation (NAF)- Research grant to FC. 


\section{References}

Altamura, S., and Muckenthaler, M. U. (2009). Iron toxicity in diseases of aging: Alzheimer's disease, Parkinson's disease and atherosclerosis. J. Alzheimers Dis. 16, 879-895. doi: 10.3233/JAD-2009-1010

Bishop, G. M., Dang, T. N., Dringen, R., and Robinson, S. R. (2011). Accumulation of non-transferrin-bound iron by neurons, astrocytes and microglia. Neurotox. Res. 19, 443-451. doi: 10.1007/s12640-010-9195-x

Bradbury, M. W. (1997). Transport of iron in the blood-brain-cerebrospinal fluid system. J. Neurochem. 69, 443-454. doi: 10.1046/j.1471-4159.1997.69 020443.x

Breuer, W., Hershko, C., and Cabantchik, Z. I. (2000). The importance of nontransferrin bound iron in disorders of iron metabolism. Transfus. Sci. 23, 185-192. doi: 10.1016/s0955-3886(00)00087-4

Brissot, P., Ropert, M., Le Lan, C., and Loréal, O. (2012). Non-transferrin bound iron: a key role in iron overload and iron toxicity. Biochim. Biophys. Acta 1820, 403-410. doi: 10.1016/j.bbagen.2011.07.014

Cheah, J. H., Kim, S. F., Hester, L. D., Clancy, K. W., Patterson, S. E. 3rd, Papadopoulos, V., et al. (2006). NMDA receptor-nitric oxide transmission mediates neuronal iron homeostasis via the GTPase Dexras1. Neuron 51, 431-440. doi: 10.1016/j.neuron.2006.07.011

Chen, Y., Khan, R. S., Cwanger, A., Song, Y., Steenstra, C., Bang, S., et al. (2013). Dexras1, a small GTPase, is required for glutamate-NMDA neurotoxicity. J. Neurosci. 33, 3582-3587. doi: 10.1523/JNEUROSCI.1497-12.2013

Crichton, R. R., and Charloteaux-Wauters, M. (1987). Iron transport and storage. Eur. J. Biochem. 164, 485-506. doi: 10.1111/j.1432-1033.1987. tb11155.x

Crichton, R. R., Dexter, D. T., and Ward, R. J. (2011). Brain iron metabolism and its perturbation in neurological diseases. J. Neural Transm. 118, 301-314. doi: 10.1007/s00702-010-0470-z

Dixon, S. J., Lemberg, K. M., Lamprecht, M. R., Skouta, R., Zaitsev, E. M., Gleason, C. E., et al. (2012). Ferroptosis: an iron-dependent form of nonapoptotic cell death. Cell 149, 1060-1072. doi: 10.1016/j.cell.2012.03.042

Du, F., Qian, Z. M., Zhu, L., Wu, X. M., Yung, W. H., Tsim, T. Y., et al. (2009). L-DOPA neurotoxicity is mediated by up-regulation of DMT1-IRE expression. PLoS One 4:e4593. doi: 10.1371/journal.pone.0004593

Gaasch, J. A., Geldenhuys, W. J., Lockman, P. R., Allen, D. D., and Van der Schyf, C. J. (2007). Voltage-gated calcium channels provide an alternate route for iron uptake in neuronal cell cultures. Neurochem. Res. 32, 1686-1693. doi: 10. 1007/s11064-007-9313-1

Gunshin, H., Mackenzie, B., Berger, U. V., Gunshin, Y., Romero, M. F., Boron, W. F., et al. (1997). Cloning and characterization of a mammalian proton-coupled metal-ion transporter. Nature 388, 482-488. doi: 10.1038/ 41343

Haeger, P., Alvarez, A., Leal, N., Adasme, T., Núñez, M. T., and Hidalgo, C. (2010). Increased hippocampal expression of the divalent metal transporter 1 (DMT1) mRNA variants $1 \mathrm{~B}$ and +IRE and DMT1 protein after NMDAreceptor stimulation or spatial memory training. Neurotox. Res. 17, 238-247. doi: $10.1007 / \mathrm{s} 12640-009-9096-\mathrm{Z}$

Halliwell, B. (2006). Oxidative stress and neurodegeneration: where are we now? J. Neurochem. 97, 1634-1658. doi: 10.1111/j.1471-4159.2006.03907.x

Hentze, M. W., Muckenthaler, M. U., Galy, B., and Camaschella, C. (2010). Two to tango: regulation of Mammalian iron metabolism. Cell 142, 24-38. doi: 10. 1016/j.cell.2010.06.028

Hershko, C., Graham, G., Bates, G. W., and Rachmilewitz, E. A. (1978). Nonspecific serum iron in thalassaemia: an abnormal serum iron fraction of potential toxicity. Br. J. Haematol. 40, 255-263. doi: 10.1111/j.1365-2141.1978. tb03662.x

Hider, R. C. (2002). Nature of nontransferrin-bound iron. Eur. J. Clin. Invest. 32(Suppl. 1), 50-54. doi: 10.1046/j.1365-2362.2002.0320s1050.x

Hozumi, I., Hasegawa, T., Honda, A., Ozawa, K., Hayashi, Y., Hashimoto, K., et al. (2011). Patterns of levels of biological metals in CSF differ among neurodegenerative diseases. J. Neurol. Sci. 303, 95-99. doi: 10.1016/j.jns.2011. 01.003

Huang, E., Ong, W. Y., Go, M. L., and Connor, J. R. (2006). Upregulation of iron regulatory proteins and divalent metal transporter-1 isoforms in the rat hippocampus after kainate induced neuronal injury. Exp. Brain Res. 170, 376-386. doi: 10.1007/s00221-005-0220-x
Hubert, N., and Hentze, M. W. (2002). Previously uncharacterized isoforms of divalent metal transporter (DMT)-1: implications for regulation and cellular function. Proc. Natl. Acad. Sci. U S A 99, 12345-12350. doi: 10.1073/pnas. 192423399

Janssen, M. C., and Swinkels, D. W. (2009). Hereditary haemochromatosis. Best Pract. Res. Clin. Gastroenterol. 23, 171-183. doi: 10.1016/j.bpg.2009. 02.004

Jeong, S. Y., and David, S. (2006). Age-related changes in iron homeostasis and cell death in the cerebellum of ceruloplasmin-deficient mice. J. Neurosci. 26, 9810-9819. doi: 10.1523/jneurosci.2922-06.2006

Ke, Y., Ho, K., Du, J., Zhu, L., Xu, Y., Wang, Q., et al. (2006). Role of soluble ceruloplasmin in iron uptake by midbrain and hippocampus neurons. J. Cell Biochem. 98, 912-919. doi: 10.1002/jcb.20740

Lane, D. J., Robinson, S. R., Czerwinska, H., Bishop, G. M., and Lawen, A. (2010). Two routes of iron accumulation in astrocytes: ascorbate-dependent ferrous iron uptake via the divalent metal transporter (DMT1) plus an independent route for ferric iron. Biochem. J. 432, 123-132. doi: 10.1042/BJ20 101317

Lopin, K. V., Gray, I. P., Obejero-Paz, C. A., Thévenod, F., and Jones, S. W. (2012). Fe $\mathrm{F}^{2+}$ block and permeation of CaV3.1 $(\alpha 1 \mathrm{G})$ T-type calcium channels: candidate mechanism for non-transferrin-mediated $\mathrm{Fe}^{2+}$ influx. Mol. Pharmacol. 82, 1194-1204. doi: 10.1124/mol.112.080184

Macco, R., Pelizzoni, I., Consonni, A., Vitali, I., Giacalone, G., Martinelli Boneschi, F., et al. (2013). Astrocytes acquire resistance to iron-dependent oxidative stress upon proinflammatory activation. J. Neuroinflammation 10:130. doi: 10. 1186/1742-2094-10-130

Mackenzie, B., Takanaga, H., Hubert, N., Rolfs, A., and Hediger, M. A. (2007). Functional properties of multiple isoforms of human divalent metal-ion transporter 1 (DMT1). Biochem. J. 403, 59-69. doi: 10.1042/bj20061290

Mizuno, S., Mihara, T., Miyaoka, T., Inagaki, T., and Horiguchi, J. (2005). CSF iron, ferritin and transferrin levels in restless legs syndrome. J. Sleep Res. 14, 43-47. doi: 10.1111/j.1365-2869.2004.00403.x

Molina, J. A., Jiménez-Jiménez, F. J., Aguilar, M. V., Meseguer, I., MateosVega, C. J., González-Muñoz, M. J., et al. (1998). Cerebrospinal fluid levels of transition metals in patients with Alzheimer's disease. J. Neural Transm. 105, 479-488. doi: 10.1007/s007020050071

Moos, T., and Morgan, E. H. (1998). Evidence for low molecular weight, nontransferrin-bound iron in rat brain and cerebrospinal fluid. J. Neurosci. Res. 54, 486-494. doi: 10.1002/(sici)1097-4547(19981115)54:4<486::aid-jnr6>3.0. $\mathrm{co} ; 2-\mathrm{i}$

Moos, T., and Morgan, E. H. (2004). The significance of the mutated divalent metal transporter (DMT1) on iron transport into the Belgrade rat brain. J. Neurochem. 88, 233-245. doi: 10.1046/j.1471-4159.2003.02142.x

Moos, T., Rosengren Nielsen, T., Skjørringe, T., and Morgan, E. H. (2007). Iron trafficking inside the brain. J. Neurochem. 103, 1730-1740. doi: 10.1111/j.14714159.2007.04976.x

Muñoz, P., Humeres, A., Elgueta, C., Kirkwood, A., Hidalgo, C., and Núñez, M. T. (2011). Iron mediates N-methyl-D-aspartate receptor-dependent stimulation of calcium-induced pathways and hippocampal synaptic plasticity. J. Biol. Chem. 286, 13382-13392. doi: 10.1074/jbc.m110.213785

Oudit, G. Y., Sun, H., Trivieri, M. G., Koch, S. E., Dawood, F., Ackerley, C., et al. (2003). L-type Ca2+ channels provide a major pathway for iron entry into cardiomyocytes in iron-overload cardiomyopathy. Nat. Med. 9, 1187-1194. doi: 10.1038/nm920

Oudit, G. Y., Trivieri, M. G., Khaper, N., Liu, P. P., and Backx, P. H. (2006). Role of L-type Ca2+ channels in iron transport and iron-overload cardiomyopathy. J. Mol. Med. (Berl.) 84, 349-364. doi: 10.1007/s00109-005-0029-x

Palmer, M. J., Hull, C., Vigh, J., and von Gersdorff, H. (2003). Synaptic cleft acidification and modulation of short-term depression by exocytosed protons in retinal bipolar cells. J. Neurosci. 23, 11332-11341.

Pelizzoni, I., Macco, R., Morini, M. F., Zacchetti, D., Grohovaz, F., and Codazzi, F. (2011). Iron handling in hippocampal neurons: activity-dependent iron entry and mitochondria-mediated neurotoxicity. Aging Cell. 10, 172-183. doi: 10. 1111/j.1474-9726.2010.00652.x

Pelizzoni, I., Macco, R., Zacchetti, D., Grohovaz, F., and Codazzi, F. (2008). Iron and calcium in the central nervous system: a close relationship in health and sickness. Biochem. Soc. Trans. 36, 1309-1312. doi: 10.1042/BST03 61309 
Pelizzoni, I., Zacchetti, D., Campanella, A., Grohovaz, F., and Codazzi, F. (2013). Iron uptake in quiescent and inflammation-activated astrocytes: a potentially neuroprotective control of iron burden. Biochim. Biophys. Acta 1832, 1326-1333. doi: 10.1016/j.bbadis.2013.04.007

Pelizzoni, I., Zacchetti, D., Smith, C. P., Grohovaz, F., and Codazzi, F. (2012). Expression of divalent metal transporter 1 in primary hippocampal neurons: reconsidering its role in non-transferrin-bound iron influx. J. Neurochem. 120, 269-278. doi: 10.1111/j.1471-4159.2011.07578.x

Rathore, K. I., Redensek, A., and David, S. (2012). Iron homeostasis in astrocytes and microglia is differentially regulated by TNF-alpha and TGF- $\beta 1$. Glia 60, 738-750. doi: 10.1002/glia.22303

Russo, N., Edwards, M., Andrews, T., O’Brien, M., and Bhatia, K. P. (2004). Hereditary haemochromatosis is unlikely to cause movement disorders-a critical review. J. Neurol. 251, 849-852. doi: 10.1007/s00415-004-0445-9

Salazar, J., Mena, N., Hunot, S., Prigent, A., Alvarez-Fischer, D., Arredondo, M., et al. (2008). Divalent metal transporter 1 (DMT1) contributes to neurodegeneration in animal models of parkinson's disease. Proc. Natl. Acad. Sci. U S A 105, 18578-18583. doi: 10.1073/pnas.0804373105

Saura, J. (2007). Microglial cells in astroglial cultures: a cautionary note. J. Neuroinflammation 4:26. doi: 10.1186/1742-2094-4-26

Semtner, M., Schaefer, M., Pinkenburg, O., and Plant, T. D. (2007). Potentiation of TRPC5 by protons. J. Biol. Chem. 282, 33868-33878. doi: 10.1074/jbc. m702577200

Tulpule, K., Robinson, S. R., Bishop, G. M., and Dringen, R. (2010). Uptake of ferrous iron by cultured rat astrocytes. J. Neurosci. Res. 88, 563-571. doi: 10. 1002/jnr.22217

Urrutia, P., Aguirre, P., Esparza, A., Tapia, V., Mena, N. P., Arredondo, M., et al. (2013). Inflammation alters the expression of DMT1, FPN1 and hepcidin and causes iron accumulation in central nervous system cells. J. Neurochem. 126, 541-549. doi: 10.1111/jnc. 12244
Wang, C. Y., and Knutson, M. D. (2013). Hepatocyte divalent metal-ion transporter-1 is dispensable for hepatic iron accumulation and non-transferrinbound iron uptake in mice. Hepatology 58, 788-798. doi: 10.1002/hep.26401

Wang, D., Wang, L. H., Zhao, Y., Lu, Y. P., and Zhu, L. (2010). Hypoxia regulates the ferrous iron uptake and reactive oxygen species level via divalent metal transporter 1 (DMT1) Exon1B by hypoxia-inducible factor-1. IUBMB Life. 62, 629-636. doi: 10.1002/iub.363

Xiong, Z. G., Pignataro, G., Li, M., Chang, S. Y., and Simon, R. P. (2008). Acidsensing ion channels (ASICs) as pharmacological targets for neurodegenerative diseases. Curr. Opin. Pharmacol. 8, 25-32. doi: 10.1016/j.coph.2007.09.001

Yanatori, I., Tabuchi, M., Kawai, Y., Yasui, Y., Akagi, R., and Kishi, F. (2010). Heme and non-heme iron transporters in non-polarized and polarized cells. BMC Cell Biol. 11:39. doi: 10.1186/1471-2121-11-39

Zarruk, J. G., Berard, J. L., Passos Dos Santos, R., Kroner, A., Lee, J., Arosio, P., et al. (2015). Expression of iron homeostasis proteins in the spinal cord in experimental autoimmune encephalomyelitis and their implications for iron accumulation. Neurobiol. Dis. doi: 10.1016/j.nbd.2015.02.001 [Epub ahead of print].

Conflict of Interest Statement: The authors declare that the research was conducted in the absence of any commercial or financial relationships that could be construed as a potential conflict of interest.

Copyright (C) 2015 Codazzi, Pelizzoni, Zacchetti and Grohovaz. This is an openaccess article distributed under the terms of the Creative Commons Attribution License (CC BY). The use, distribution and reproduction in other forums is permitted, provided the original author(s) or licensor are credited and that the original publication in this journal is cited, in accordance with accepted academic practice. No use, distribution or reproduction is permitted which does not comply with these terms. 